\title{
Molecular typing and drug sensitivity testing of Mycobacterium tuberculosis isolated by a community-based survey in Ethiopia
}

Muluwork Getahun 1*, Gobena Ameni², Abebaw Kebede', Zelalem Yaregal', Elena Hailu³, Grimay Medihn², Daniel Demssie ${ }^{1}$, Feven Girmachew ${ }^{1}$, Yetnebersh Fiseha' ${ }^{1}$ Abyot Meaza ${ }^{1}$, Nathneal Dirse ${ }^{1}$, Mulualem Agonafir ${ }^{1}$, Feleke Dana', Fasil Tsegaye ${ }^{1}$, Zeleke Alebachew ${ }^{1}$, Almaz Abebe ${ }^{1}$, Amha Kebede $^{1}$ and Eshetu Lemma ${ }^{1}$

\begin{abstract}
Background: The identification of circulating TB strains in the community and drug sensitivity patterns is essential for the tuberculosis control program. This study was undertaken to identify M. tuberculosis strains circulating in selected communities in Ethiopia as well as to evaluate the drug sensitivity pattern of these strains.

Method: This study was a continuation of the Ethiopian National TB Prevalence Survey that was conducted between 2010 and 2011. Culture-positive isolates of M. tuberculosis from previous study were typed using region of difference (RD) 9-based polymerase chain reaction (PCR) and spoligotyping. Drug sensitivity testing was conducted using the indirect proportion method on Lowenstein-Jensen media.

Result: All 92 isolates were confirmed as M. tuberculosis by RD9-based PCR and spoligotyping of 91 of these isolates leds to the identification of 41 spoligotype patterns. Spoligotype revealed higher diversity (45\%) and among this $65.8 \%(27 / 41)$ were not previously reported. The strains were grouped into 14 clusters consisting of 2-15 isolates. The dominant strains were SIT53, SIT149 and SIT37 consisting of 15, 11, and 9 isolates, respectively. Our study reveals $70 \%(64 / 91)$ clustered strains and only $39.1 \%$ (25/64) occurred within the same Kebele. Further assignment of the strains to the lineages showed that $74.7 \%$ (68/91) belonged to Euro-American lineage, $18.6 \%$ $(17 / 91)$ to East Africa Indian lineage and the remaining $6.5 \%$ (6/91) belonged to Indo-oceanic lineage. Valid drug susceptibility test results were available for 90 of the 92 isolates. Mono-resistance was observed in $27.7 \%$ (25/90) and poly-resistance in $5.5 \%$ (5/90) of the isolates. Moreover, multi-drug resistance (MDR-TB) was detected in $4.4 \%$ of the isolates whilst the rest (60/90) were susceptible to all drugs. The highest level of mono-resistance, $26.6 \%$ (24/90), was observed for streptomycin with majority (91.1\%) of streptomycin mono-resistant strains belonging to the Euro-American lineage.
\end{abstract}

Conclusion: In this study, the strains of M. tuberculosis circulating in selected sites of Ethiopia were identified along with the drug sensitivity patterns. Thus, these findings are useful for the TB Control Program of the country.

\footnotetext{
* Correspondence: mimishaget@yahoo.com

'Ethiopian Public Health Institute, Addis Ababa, Ethiopia

Full list of author information is available at the end of the article
} 


\section{Background}

There are an estimated 9 million new cases of TB every year. TB is the second leading cause of death from an infectious disease worldwide and is responsible for 1.5 million deaths annually. Control of TB is becoming a challenge to the endemic countries due to the emergence of drug resistance. Recently, the WHO Global TB Report estimated that total of 480,000 people developed MDR-TB worldwide in 2013. The prevalence of MDR-TB has been estimated to be $3.5 \%$ in newly diagnosed cases and $20.5 \%$ in previously treated TB cases [1].

In Ethiopia, TB is a major public health problem and the WHO categorizes Ethiopia as a high TB and MDR-TB burden country [1]. The first Ethiopian National TB prevalence survey was conducted between 2010 and 2011. The prevalence of smear-positive TB among persons aged $\geq 15$ years was 108/100,000 (95\% CI 73-143), whereas the prevalence of bacteriologically confirmed TB in the same age group was 277/100,000 (95\% CI 208-347) [2]. According to the nationwide drug resistance survey conducted between 2003 and 2005 the overall prevalence of MDR-TB was $2.5 \%$. The prevalence of MDR-TB was $1.6 \%$ and $11.8 \%$ in newly diagnosed TB cases and previously treated cases, respectively [3].

Knowledge on the Mycobacterium tuberculosis (M. tuberculosis) strains circulating in Ethiopian communities and evaluation of the drug sensitivity patterns of these strains is useful for the TB control program in the country. Since the study was done on positive cultures isolated from community-based survey, those patients who have low health seeking behavior were mostly captured. Therefore, this study was undertaken for the identification of the $M$. tuberculosis strains circulating in selected communities in Ethiopia and thereafter to evaluate the drug sensitivity pattern of these strains.

\section{Methods \\ Study population}

This study was a continuation of the Ethiopian National TB Prevalence Survey that was conducted between 2010 and 2011. The participants for the prevalence survey carried out by Kebede $\mathrm{AH}$ and colleagues were recruited from 85 Kebeles (communes), selected from 85 Woredas (districts) which were stratified as urban, rural and pastoralist population. The number of Kebeles covered in rural, urban and pastoralist areas were 63, 14 and 8 respectively. Sample size determination for the prevalence survey is reported elsewhere [2]. The planned sample size for prevalence survey was 46,514 adults aged $\geq 15$ years. Following this 85 Woredas were selected by multistage cluster sampling using probability proportion to size (PPS) from three strata (urban, rural and pastoralist). In each selected districts one Kebele with estimated eligible adult of 548 was selected using PPS. However during the recruitment of participants the total numbers of individuals from 85 selected clusters were 51,667. Since cluster sampling was used and to maximize sample size, all eligible individuals were invited to participate in the study. Of the total eligible individuals, 46,697 (90 \%) participated in the study. The case enrollments for the study were based on symptoms suggestive of TB (cough $\geq 2$ weeks) and/or any abnormality in the lung detected using chest X-ray. For individuals exempt from chest X-ray who did not have cough $\geq 2$ weeks, one of the following criteria was used: weight loss $>3 \mathrm{~kg}$ in the last 1 month, night sweats $>2$ weeks, fever $>2$ weeks and contact with a TB patient in the last year. Using the above criteria a total of 6080 (13\%) participants were eligible for sputum examination. Of these, 5868 (97\%) submitted at least one sputum specimen and 5606 (92\%) submitted two specimens [2]. The present study was carried out on culture positive isolates identified from the prevalence survey.

\section{Spatial distribution of the study sites at Woreda level}

The spatial distributions of the selected study sites are displayed at Woredas level (Fig. 1).

\section{Laboratory tests}

The laboratory analysis was carried out from July to December 2012. For this study, deletion typing, spoloigotyping and drug susceptibility testing were done on positive isolates. Culture and identification of the isolates for the prevalence survey were performed using $4 \%$ $\mathrm{NaOH}$ and Capilia test respectively.

\section{Culture and identification}

Equal amounts of $4 \% \mathrm{NaOH}$ were added to sputum in a $50 \mathrm{ml}$ screw capped tube. The mixture was vortexed for 1-2 min and kept for $15 \mathrm{~min}$ at room temperature. Sterile phosphate buffer ( $\mathrm{pH}=6.8$ ) was added up to $45 \mathrm{ml}$ and then centrifuged at $4{ }^{\circ} \mathrm{C}$ for $15 \mathrm{~min}$ at $3000 \mathrm{~g}$. After decanting the supernatant, a few drops of sterile phosphate buffer ( $\mathrm{pH}=6.8$ ) was added to reconstitute the sediment. An aliquot of 100-200 $\mu \mathrm{l}$ of the sample sediment was then inoculated into two bottles of Löwenstein- Jensen (LJ) media. The inoculated media was incubated at $37{ }^{\circ} \mathrm{C}$. Bacterial growth was checked for contamination and fast growers in first week and followed weekly up to 8 weeks. Cultures with no growth until the eighth weeks were recorded as negative [4]. Species identification was done for positive LJ cultures. Identification of $M$. tuberculosis complex from other mycobacterial species was performed using Capilia test. The test is based on the M. tuberculosis antigen MPB64, which is specific to the members of the $M$. tuberculosis complex. Testing and interpretation of the assays was done according to the manufacturer instruction [5]. 


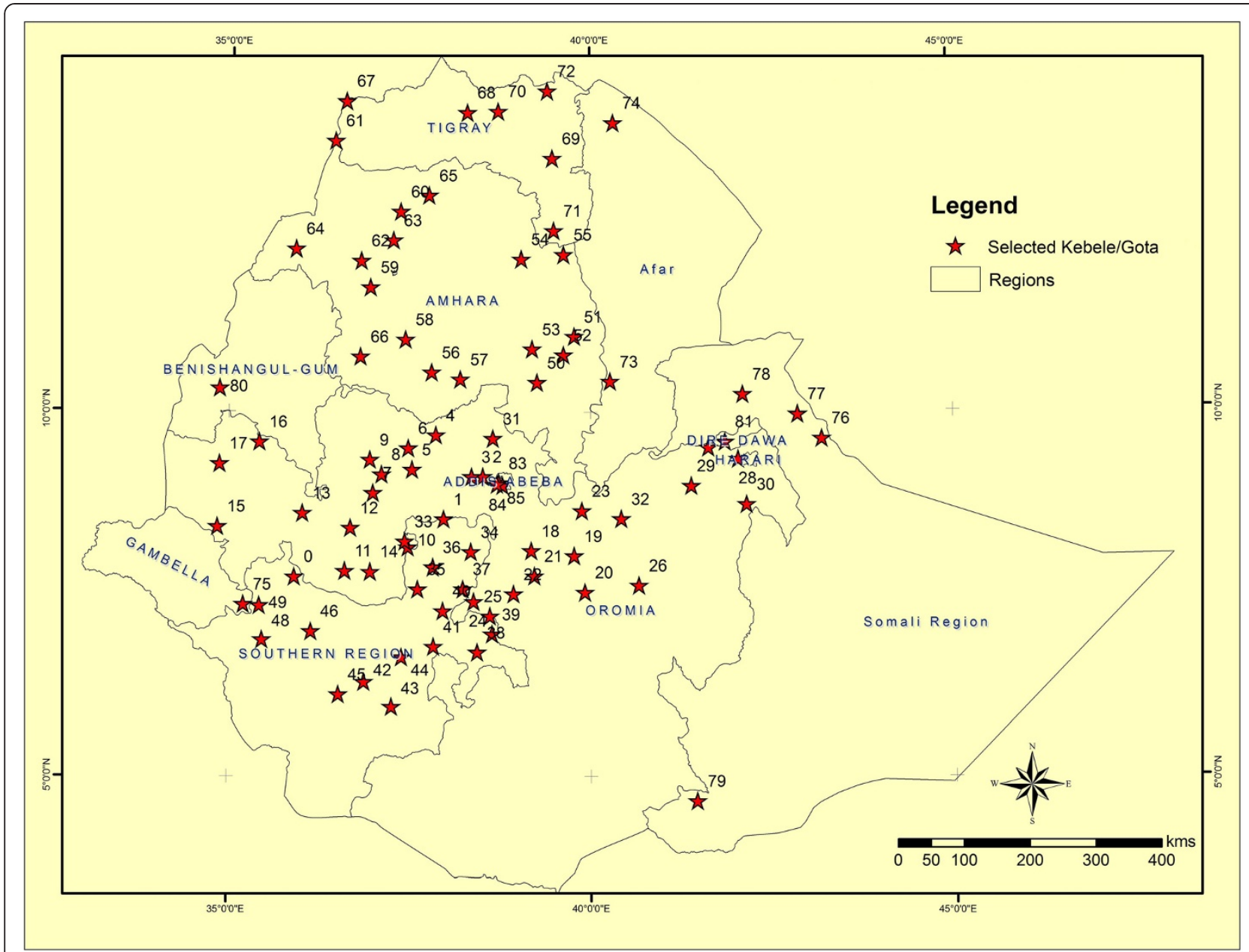

Fig. 1 Spatial distribution study sites at Woreda level

\section{Molecular characterization of the isolates Identification}

Identification of $M$. tuberculosis from the other members of the $M$. tuberculosis complex species was done using region of difference (RD)-9 polymerase chain reaction. RD9PCRdeletion typing was performed on heat-killed cells to confirm the presence or absence of RD9 using three primers RD9flankF, RD9IntR and RD9flankR [6]. PCR amplification was done as indicated by Berg et al. using a commercially available kit (Qiagen, United Kingdom). Interpretation of the result was based on differences in molecular weight. A molecular weight of 396bp was considered as M. tuberculosis or $M$. canettii, while a molecular weight 575bp was considered M. bovis or M. africanum [7].

\section{Molecular typing}

Strain typing of $M$. tuberculosis was done using spoligotyping as previously described by Kamerbeek and colleagues [8]. The PCR amplification of DNA was done by targeting the direct repeat (DR) locus, the region with the highest level of polymorphism in the M. tuberculosis chromosome. The DR region was amplified using primers DRa (GGTTTTGGGTCTGACGAC) and DRb (CCGA GAGGGGACGGAAAC). Spoligotyping was performed with a commercially available kit according to the manufacturer instructions (Isogen Bioscience BV Maarssen, The Netherlands). Lineage and SIT (Shared International Types) number assignment was done using SPOLD4 and SPOTCLUST [9].

\section{Drug Susceptibility testing}

Drug susceptibility testing (DST) was performed for first line drugs; isoniazid, streptomycin, rifampicin and ethambutol, using indirect proportion method on Lowenstein-Jense media. The critical concentration for each drug was $0.2 \mu \mathrm{g} / \mathrm{ml}, 4 \mu \mathrm{g} / \mathrm{ml}, 40 \mu \mathrm{g} / \mathrm{ml}$ and $2 \mu \mathrm{g} / \mathrm{ml}$ for isoniazid, streptomycin, rifampicin and ethambutol respectively. Result interpretation was done by comparing growth on control media and media containing drug. If more than $1 \%$ of the test population was observed on the drug containing media, the result was interpreted as resistant to that drug [10] 


\section{Statistical analysis}

Statistical analysis was carried out using SPSS software packages. Patient characteristics for differences in proportions were compared. The chi square test was used to assess statistical significant difference. $P$ values of 0.05 or less were regarded as significant.

\section{Ethical consideration}

The laboratory analysis was done on stored culture positive samples. Specific patient identifiers were not used for this study. Ethical clearance was obtained from Scientific and Ethics Review Committee at Ethiopian Public Health Institute.

\section{Result}

\section{Background characteristics}

The mean age of culture positive participants was 34.4 years (95\% CI 31.2-37.7). The overall culture positivity rate was $1.6 \%$ (96/5863). The distribution of culture positivity across the categories of background characteristics of TB suspects is summarized in Table 1. The culture positivity was significantly associated with age and region of the study participants. High culture positivity rate $(3.2 \%)$ was observed in age group between 15 and 24 . Among three regions with high culture positivity rate (Addis Ababa, SNNPR and Gambela) the contribution of younger age categories (15-34) was $44 \%$ (4 out of 9), $60 \%$ (18 out of 30 ) and $80 \%$ (4 out of 5) from the total positive culture from those regions.

\section{Molecular characterization of the isolates Identification}

Out of 96 isolates positive for M. tuberculosis complex based on Capilia TB immunochromographic assay, 4 samples were excluded from RD9-PCR analysis (2 isolates failed to grow after re-culturing and 2 cultures were not retrieved). The remaining 92 isolates were analyzed based on region of difference analysis. RD9 analysis showed that 92 isolates contained an intact RD9. This indicated that the 92 isolates were Mycobacterium tuberculosis species or M. canettii.

\section{Molecular typing}

Ninety two isolates were characterized using spoligotyping and 91 of them showed good patterns. Spoligotyping identified 41 different spoligotypes with the overall diversity of $45 \%$ and among this $65.8 \%$ (27/41) were not previously reported. The isolates were grouped into 14 clusters consisting of 2-15 isolates each. The dominant strains of $M$. tuberculosis were SIT53 (15/91), SIT149 (11/91) and SIT37 (9/91). Our study reveals 70 \% (64/ 91) clustered strains of which 53 were registered in the international database whereas 11 were newly identified.

Table 1 Culture result Vs sex, age, region and resident type

\begin{tabular}{|c|c|c|c|c|c|c|}
\hline & & Culture Positive & Culture Negative/NTM/Contaminated & Total & Culture Positivity Rate & $P$-value \\
\hline \multirow[t]{2}{*}{ Sex } & Male & 51 & 3122 & 3173 & 1.6 & 0.844 \\
\hline & Female & 45 & 2645 & 2690 & 1.7 & \\
\hline \multirow[t]{6}{*}{ Age } & $15-24$ & 32 & 962 & 994 & 3.2 & $<0.001$ \\
\hline & $25-34$ & 21 & 1183 & 1204 & 1.7 & \\
\hline & $35-44$ & 17 & 1084 & 1101 & 1.5 & \\
\hline & $45-54$ & 12 & 992 & 1004 & 1.2 & \\
\hline & $55-64$ & 10 & 736 & 746 & 1.3 & \\
\hline & $>=65$ & 4 & 810 & 814 & 0.5 & \\
\hline \multirow[t]{10}{*}{ Region } & Oromiya & 28 & 2250 & 2278 & 1.2 & $<0.001$ \\
\hline & SNNPR & 30 & 1191 & 1221 & 2.5 & \\
\hline & Amhara & 10 & 948 & 958 & 1 & \\
\hline & Tigray & 6 & 505 & 511 & 1.2 & \\
\hline & Afar & 1 & 143 & 144 & 0.7 & \\
\hline & Gambela & 5 & 83 & 88 & 5.7 & \\
\hline & Somali & 7 & 388 & 395 & 1.8 & \\
\hline & Benshangul & 0 & 25 & 25 & 0 & \\
\hline & Dire Dawa & 0 & 45 & 45 & 0 & \\
\hline & Addis Ababa & 9 & 189 & 198 & 4.5 & \\
\hline \multirow[t]{3}{*}{ Resident } & Urban & 14 & 744 & 758 & 1.8 & 0.844 \\
\hline & Rural & 72 & 4349 & 4421 & 1.6 & \\
\hline & Pastoral & 10 & 674 & 684 & 1.5 & \\
\hline
\end{tabular}


Table 2 Distribution of previously registered and newly identified cluster in Kebeles

\begin{tabular}{lcc}
\hline SIT for previously registered strains & $\begin{array}{c}\text { Same Kebele } \\
\text { Number }(\%)\end{array}$ & $\begin{array}{c}\text { Different Kebele } \\
\text { Number (\%) }\end{array}$ \\
\hline 53 & $4(26.7)$ & $11(73.3)$ \\
149 & $4(36.4)$ & $7(63.6)$ \\
37 & $4(44.4)$ & $5(55.6)$ \\
26 & $0(0.0)$ & $3(100)$ \\
119 & $0(0.0)$ & $3(100)$ \\
910 & $0(0.0)$ & $3(100)$ \\
247 & $2(66.7)$ & $1(33.3)$ \\
21 & $0(0.0)$ & $2(100)$ \\
289 & $0(0.0)$ & $2(100)$ \\
777 & $2(100)$ & $0(0.0)$ \\
Sub Total & $16(30.2)$ & $37(69.8)$ \\
Newly identified strains & & \\
Orphan & $2(50.0)$ & $2(50.0)$ \\
Orphan & $3(100)$ & $0(0.0)$ \\
Orphan & $2(100)$ & $0(0.0)$ \\
Orphan & $2(100)$ & $0(0.0)$ \\
Sub Total & $9(81.8)$ & $2(18.2)$ \\
Total & $25(39.1 \%)$ & $39(60.9 \%)$ \\
\hline
\end{tabular}

Cluster formation within Kebeles was observed only $39.1 \%(25 / 64)$ (Table 2). The proportion of Kebeles having cluster isolates within the same Kebeles in urban, rural and pastoralist were $28.5 \%(4 / 14), 13.4 \%(9 / 67)$ and $14.2 \%(1 / 7)$ respectively. Geographic Information System (GIS) mapping of cluster position of the strain showed that clustering location was far apart (Fig. 2). Among 27 single strains $40.7 \%$ were registered in the international database. Further assignment of the strains to the lineages showed that $74.7 \%$ (68/91) belonged to Euro-American lineage, $18.6 \%$ (17/91) belonged to East Africa Indian lineage and $6.5 \%(6 / 91)$ to Indo-oceanic lineage.

\section{Drug susceptibility test (DST)}

Valid DST results for all first line anti-TB drugs were available for 90 isolates (Table 3). Two isolates were excluded because of contamination. The drug susceptibility pattern of the isolates revealed that $66.6 \%(60 / 90)$ of the isolates were susceptible to the four first-line drugs tested (streptomycin, isoniazid, rifampicin and ethambutol). Mono-resistance to any one of the four first line drugs tested was found in $27.7 \%$ (25/90) of the cases and polyresistance was found in $5.5 \%$ (5/90) of cases. The highest level of mono-resistance was observed for streptomycin $26.6 \%$ (24/90). Of the 24 streptomycin mono-resistant strain majority $(91.1 \%)$ belonged to the Euro-American lineage and the rest belongs to Indo-oceanic lineage.
Multi-drug resistance (MDR) was detected in $4.4 \%(4 / 90)$ of the cases. The overall MDR-TB prevalence in national survey conducted between 2003 and 2005 was $2.5 \%$ [2]. The prevalence of MDR-TB did not show any significant increase compared to the first round drug resistance survey conducted between 2003 and $2005(P=0.287)$. Among the four MDR-TB detected, three of them were isolated from young patients (age ranges 16-20). The lineage of those MDR TB cases showed that three of them belonged to East Africa Indian lineage and one belonged to the Euro-American lineage.

\section{Discussion}

Tuberculosis (TB) is the most common opportunistic infection in HIV positive patients and TB can occur at any time during the course of HIV infection [11]. This study's findings showed high culture positivity among the younger age groups and in three regions. The TB/ HIV sentinel report from 2010 to 2014 indicated that out of those three regions, two regions ranked as the two most HIV prevalent among TB patients (unpublished data), which may account for the higher culture positivity rate in younger age groups in those regions.

Most of human TB is caused by $M$. tuberculosis although some cases are caused by M. bovis. In this study, RD9deletion typing was used to differentiate between $M$. bovis and $M$. tuberculosis [12, 13]. Even though most of the isolates were collected in rural and pastoralist population where raw milk consumption is widely practiced, all the isolates obtained by this study were M. tuberculosis. The absence of $M$. bovis among the isolates collected from rural communities was unexpected and contradicts with the earlier studies $[14,15]$. The $\mathrm{r}$ absence of $M$. bovis requires further study and may be due to the manifestation of $M$. bovis in extra-pulmonary TB.

Cluster formation and epidemiological links have been used to qualify recent transmission [16]. Our finding reveals clustering within same Kebele was low (39.1\%) even without considering the parameters for making an epidemiological link. Since the larger portions of our samples were collected from rural populations that were sparsely populated, this might result in low rate of transmission as a result clustering rate will be decreased, which is in line with previous findings $[16,17]$. Branden and colleagues suggested that the transmission of common endemic strains of $M$. tuberculosis occurs in relatively closed populations but in a rural setting the presence of clustering often is not associated with recent transmission; it could be the result of reactivation of remote infections acquired years or decades earlier.

Understanding the prevalence of TB drug resistance offers an opportunity to assess the extent of resistant bacteria transmission in the community as well as evaluate effect of the National Tuberculosis Program [18]. 


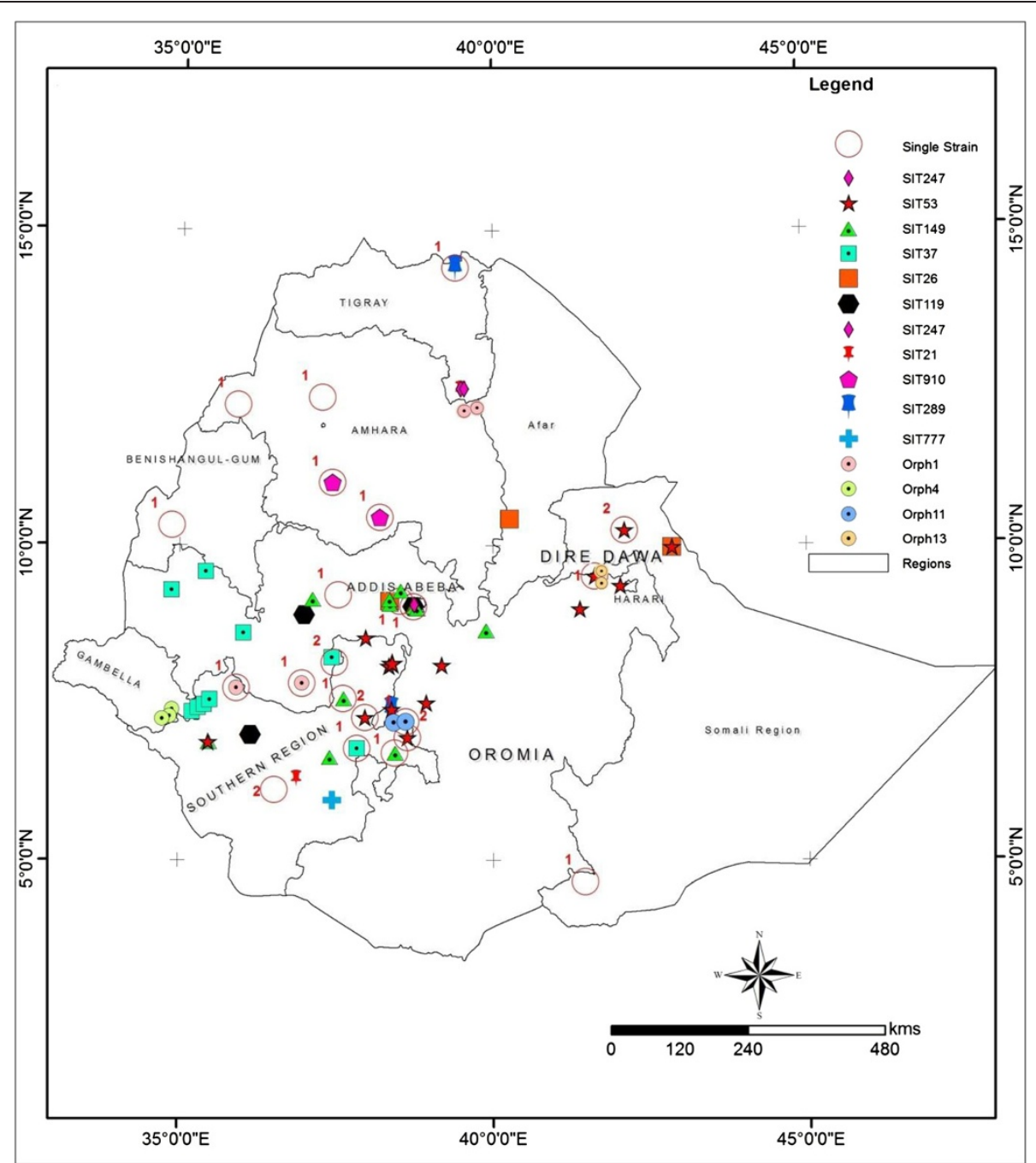

Fig. 2 Distribution of strains in their respective collection sites at Woreda level: Both registered and orphan clustered strains were indicated in colored. Orphans were given a number according to their order of identification. Both registered and orphan single isolates is represented in red hallow circle. The numeral in the side of the circle indicates that the number of single strains identified in that specific location

Table 3 Susceptibility pattern of 90 isolate for first line drugs

\begin{tabular}{llc}
\hline & Number & Percentage \\
\hline Susceptible & 60 & 66.6 \\
Mono-resistance & 25 & 27.7 \\
Resistant to & & \\
INH only & 1 & 1.1 \\
SM only & 24 & 26.6 \\
INH + SM & 1 & 1.1 \\
MDR & 4 & 4.4 \\
INH + SM + RIF & 1 & 1.1 \\
INH + SM + RIF + EMB & 3 & 3.3 \\
\hline
\end{tabular}

The MDR rate did not show any significant increment compared to the previous nationwide surveillance report even though the study population was different [3]. A study conducted in the Philippines at hospitals in Metro Manila, multi- drug resistant TB prevalence rates in hospital ranged from $14-45 \%$, whereas the community rate of MDR TB in Metro Manila was $6.4 \%$ [19]. This suggested that high rate of MDR TB occur in health facilities. Thus in Ethiopia, the rate of MDR TB in the health facilities might be higher than the finding of this current study. Out of 4 MDR cases identified in the present study, three MDR TB cases occurred in participants $16-20$ years old. As reported by another study [20] the high prevalence of primary MDR-TB in a young population group implies transmission of drug resistant strains. 


\section{Conclusions}

In this study, the strains of $M$. tuberculosis circulating in selected sites of Ethiopia were identified along with the drug sensitivity patterns. More than $83 \%$ of the population is living in rural areas of Ethiopia. Since declining incidence of $\mathrm{TB}$ is expected with decreased clustering, coupling case detection and treatment alone in those large portions of the rural population will have a huge contribution to the reduction of TB. Besides, TB and MDR rate in young was high but with such small number of cases the possible relationship is inconclusive it needs further study.

\section{Competing interests}

We declare that there are no financial, professional or personal competing interests that might have influenced the performance or presentation of the work described in this manuscript.

\section{Authors' contribution}

MG conceived, designed, carried out the study, and wrote the manuscript. GA advised in proposal development, laboratory analysis, data analysis, and manuscript write up. AK involved in data analysis and manuscripts write up. ZY carried out quality control for all laboratory analysis. EH carried out spoligotyping, GM advised in proposal development, data analysis and manuscript write up. DD, FG, YF, AM and ND carried out drug susceptibility testing. ZA, FT, MA and FD carried out data extraction and data analysis. EL, $A K$, and $A A$ advise in proposal development, data analysis and manuscript write up. All authors read and approved the final manuscript.

\section{Acknowledgment}

We would like to thank EPHI, ALIPB and AHRI for the financial support. Our acknowledgment also goes to Girma Urgeacha for doing GIS mapping, Yared Kebede, Blessing Marondera and Justin Taylor for editing the manuscript, Wegene Tamene and Aboma Zewude for their technical support.

\section{Author details}

${ }^{1}$ Ethiopian Public Health Institute, Addis Ababa, Ethiopia. ${ }^{2}$ Aklilu Lema Institute of Pathobiology, Addis Ababa, Ethiopia. ${ }^{3}$ Armauer Hansen Research Institute, Addis Ababa, Ethiopia.

Received: 27 December 2014 Accepted: 28 July 2015

Published online: 06 August 2015

References

1. World Health Organization Global Tuberculosis Report. Who, Geneva: Annual Report; 2014

2. Kebede AH, Alebachew Z, Tsegaye F, Lemma E, Abebe A, Agonafir M, et al. The first population-based national tuberculosis prevalence survey in Ethiopia, 2010-2011. Int J Tuberc Lung Dis. 2014;18:635-9.

3. World Health Organization Anti-Tuberculosis Drug Resistance in the World: Fourth Global Report; 2008

4. Kantor IND, Kim SJ, Frieden T: Laboratory Services in Tuberculosis Contro Culture Part III. World Health Organization 1998, 57-58.

5. Abe C, Hirano K, Tomiyama T. Simple and Rapid Identification of the Mycobacterium tuberculosis Complex by Immunochromatographic Assay Using Anti-MPB64 Monoclonal Antibodies. J Clin Microbiol. 1999;37:3693-7.

6. Brosch R, Gordon SV, Marmiesse MP. A new evolutionary scenario for the Mycobacterium tuberculosis complex. Proc Natl Acad Sci. 2002;99(6):3684-9.

7. Nakajima C, Rahim Z, Fukushima Y, Sugawara I, van de Zanden AGM, Tamaru A, et al. Identification of Mycobacterium tuberculosis clinical isolates in Bangladesh by a species distinguishable multiplex PCR. BioMedCenter Infect Dis. 2010;10(118):1-7.

8. Kamerbeek J, Schouls L, Kolk A, van Agterveld M, van Soolingen D, Kuijper S, et al. Simultaneous detection and strain differentiation of Mycobacterium tuberculosis for diagnosis and epidemiology. J Clin Microbiol. 1997:35(4):907-14.
9. Molhuizen HOF, Bunschoten AE, Schouls LM, van Embden J. Rapid detection and simultaneous strain differentiation of Mycobacterium tuberculosis complex bacteria by spoligotyping. Methods Mol Biol. 1998;101:381-94

10. Kent PT, and Kubica GP, Public health mycobacteriology a guide for the level III laboratory. Centers for Disease control 1985: 159-185.

11. Swaminathan S, Ramachandran R, Baskaran G, Paramasivan CN, Ramanathan $U$, Venkatesan P, et al. Risk of development of tuberculosis in HIV-infected patients. Int J Tuberc Lung Dis. 2000;4:839-44.

12. Cataldi A, Romano MI. Tuberculosis caused by Other Members of the $M$. tuberculosis Complex. In: Palomino JC, Leão SC, Ritacco V, editors. Tuberculosis 2007 From basic science to patient care. Belgium: Bernd Sebastian Kamps and Patricia Bourcillier; 2007. p. 283-314

13. Rodríguez E, Sánchez LP, Pérez S, Herrera L, Jiménez MS, Samper $S$, et al. Human tuberculosis due to Mycobacterium bovis and M. caprae in Spain, 2004-2007. Int J Tuberc Lung Dis. 2009;13(12):1536-41.

14. Regassa A, Medhin G, Ameni G. Bovine tuberculosis 302 is more prevalent in cattle owned by farmers with active tuberculosis in central Ethiopia. Elsevier. 2007;178(2008):119-25.

15. Shitaye JE, Tsegaye W, Pavlik I. Bovine tuberculosis infection in animal and human populations in Ethiopia: a review. Vet Med. 2007:52:317-32. Review Article.

16. Braden CR, Templeton GL, Cave MD, Valway S, Onorato IM, Castro KG, et al Interpretation of restriction fragment length polymorphism analysis of Mycobacterium tuberculosis isolates from a state with a large rural population. J Infect Dis. 1997;175:1446-52.

17. Ellis BA, Crawford JT, Braden CR, McNabb SJN, Moore M, Kammerer S, et al. Molecular epidemiology of tuberculosis in a sentinel surveillance population. Emerg Infect Dis. 2002;8:1197-209.

18. Paramasivan CN, Bhaskarair K, Venkataraman $\mathrm{P}$, Chandrasekaran V, Narayanan PR. Surveillance of drug resistance in tuberculosis in the state of Tamil Nadu. Indian J Tuberc. 2000;47:27-33.

19. Mendoza MT, Tan-Torres T, Ang CF, Arciaga R, Elona F, Retuta M, et al. Community-Based surveillance for drug resistance of Mycobacterium tuberculosis in selected areas in the Philippines. Phil J Microbiol Infect Dis. 2002;3:169-75

20. Merza MA, Farnia P, Tabarsi P, Khazampour M, Masjedi MR, Velayati AA. Anti tuberculosis drug resistance and associated risk factors in a tertiary level TB centre in Iran: a retrospective analysis. J Infect Dev Ctries. 2011;5(7):511-9.

\section{Submit your next manuscript to BioMed Central and take full advantage of:}

- Convenient online submission

- Thorough peer review

- No space constraints or color figure charges

- Immediate publication on acceptance

- Inclusion in PubMed, CAS, Scopus and Google Scholar

- Research which is freely available for redistribution 